\title{
SOME NEW CLASSES OF CONVEX FUNCTIONS AND INEQUALITIES
}

\author{
MUHAMMAD UZAIR AWAN, MUHAMMAD ASLAM NOOR, \\ KHALIDA INAYAT NOOR, AND AWAIS GUL KHAN
}

Received 13 December, 2016

\begin{abstract}
In this article, we introduce some new class of convex functions involving two arbitrary auxiliary functions $h_{1}, h_{2}: I \rightarrow \mathbb{R}$, which are called $\left(h_{1}, h_{2}\right)$-convex functions. We derive some new integral inequalities for these classes of convex functions. We also discuss some special cases which can be deduced from our main results. Results obtained in this paper may be viewed as a significant refinement and improvement of the previously known results. The ideas and techniques of this work may be a starting point for future research.
\end{abstract}

2000 Mathematics Subject Classification: 26D15; 26A51

Keywords: convex function, Hermite-Hadamard inequalities

\section{INTRODUCTION}

A set $\mathcal{C} \subset \mathbb{R}$ is said to be a convex set, if

$$
t x+(1-t) y \in \mathscr{C}, \quad \forall x, y \in \mathscr{C}, t \in[0,1] .
$$

A function $f: \mathcal{C} \rightarrow \mathbb{R}$ is said to be a convex function in the classical sense, if

$$
f(t x+(1-t) y) \leq t f(x)+(1-t) f(y), \quad \forall x, y \in \mathcal{C}, t \in[0,1] .
$$

In recent years several new generalizations of classical convexity have been given, for example see $[1-3,8,17,18]$. Varosanec [18] introduced the notion of $h$-convexity which along with classical convex functions generalizes several other class of convex functions. The formal definition of $h$-convex functions is given as:

Definition 1 ([18]). Let $h:(0,1) \subseteq J \rightarrow \mathbb{R}$ be a non-negative function, $h \not \equiv 0$. We say that $f: \mathcal{\ell} \rightarrow \mathbb{R}$ is an $h$-convex function, if $f$ is non-negative and for all $x, y \in \mathcal{C}$, $t \in(0,1)$, we have

$$
f(t x+(1-t) y) \leq h(t) f(x)+h(1-t) f(y) .
$$

Corresponding author: Muhammad Uzair Awan. 
For the different choices of the auxiliary function $h($.$) , we have different other$ classes of convex functions such as: Breckner type of $s$-convex functions [1], Godunova-Levin-Dragomir type of $s$-convex functions [4], Godunova-Levin type of functions [8] and $P$-functions [7]. Since the appearance of this definition many researchers shown their special interest in studying this class of convex functions. Sarikaya et al. [15] has improved the Hermite-Hadamard's inequality for this class of convex functions. Recent trend of the research in this field has shown that theory of convexity and theory of inequalities have a close relationship. Many inequalities can be obtained via convex functions and naturally they can be extended for generalizations of convex functions, see [5, 6, 10-12,14,16].

Motivated by the research going on in this field, we introduce the notion of socalled $\left(h_{1}, h_{2}\right)$-convex functions, which is the main motivation of this paper. These classes involves two auxiliary functions namely $h_{1}, h_{2}: J \rightarrow \mathbb{R}$. We show that these classes include several new and known classes of convex functions as special cases. We also derive some new estimates for Hermite-Hadamard type of inequalities and for the integral $\int_{a}^{b}(u-a)^{\alpha}(b-u)^{\beta} f(u) \mathrm{d} u$ via $\left(h_{1}, h_{2}\right)$-convex functions. Some new and known special cases which can be deduced from our main results, are also discussed.

\section{PRELIMINARIES}

We now define the new classes of convex functions involving two arbitrary functions.

Definition 2. Let $h_{1}, h_{2}:(0,1) \subseteq J \rightarrow \mathbb{R}$ be two real functions, $h_{1}, h_{2} \not \equiv 0$. We say that $f: \mathcal{C} \rightarrow \mathbb{R}$ is an $\left(h_{1}, h_{2}\right)$-convex function, if

$f(t x+(1-t) y) \leq h_{1}(t) h_{2}(1-t) f(x)+h_{1}(1-t) h_{2}(t) f(y), \quad \forall x, y \in \mathcal{C}, t \in(0,1)$.

We now discuss several special cases.

I. If $h_{2}(t)=1$, then Definition 2 reduces to the definition for $h$-convex functions [18].

II. If $h_{1}(t)=1=h_{2}(t)$, then Definition 2 reduces to the definition for $P$-functions [7].

III. If $h_{1}(t)=t^{s}$ and $h_{2}(t)=t^{s}$ in Definition 2, then we have the class of $s$-convex functions of third kind.

Definition 3. Let $s \in[0,1]$ be a real number. We say that $f: \mathcal{C} \rightarrow \mathbb{R}$ is an $s$-tgsconvex functions, if

$$
f(t x+(1-t) y) \leq t^{s}(1-t)^{s}[f(x)+f(y)], \quad \forall x, y \in \mathcal{C}, t \in[0,1] .
$$


Note that if we take $s=1$ in Definition 3, then we have the definition of $\operatorname{tg} s$-convex functions [17].

IV. If $h_{1}(t)=t^{-s}$ and $h_{2}(t)=t^{-s}$ in Definition 2, then we have the class of $s$ Godunova-Levin-Dragomir $\operatorname{tg} s$-convex functions.

Definition 4. Let $s \in(0,1]$ be a real number. We say that $f: \mathcal{C} \rightarrow \mathbb{R}$ is an $s$ Godunova-Levin-Dragomir $\operatorname{tg} s$-convex function, if

$$
f(t x+(1-t) y) \leq \frac{1}{t^{s}} \frac{1}{(1-t)^{s}}[f(x)+f(y)], \quad \forall x, y \in \mathcal{C}, t \in[0,1] .
$$

Note that if we take $s=1$ in Definition 4, then we have the definition of GodunovaLevin type of $\operatorname{tg} s$-convex functions, which appears to be a new definition.

V. If $h_{1}(t)=t^{s_{1}}$ and $h_{2}(t)=t^{s_{2}}$ in Definition 2, then we have a new class of convex functions which is called as Breckner type of $\left(s_{1}, s_{2}\right)$-convex functions.

Definition 5. Let $s_{1}, s_{2} \in(0,1]$ be two real numbers. We say that $f: \ell \rightarrow \mathbb{R}$ is an $\left(s_{1}, s_{2}\right)$-convex function, if

$$
f(t x+(1-t) y) \leq t^{s_{1}}(1-t)^{s_{2}} f(x)+(1-t)^{s_{1}} t^{s_{2}} f(y), \quad \forall x, y \in \mathcal{C}, t \in[0,1] .
$$

VI. If $h_{1}(t)=t^{-s_{1}}$ and $h_{2}(t)=t^{-s_{2}}$ in Definition 2, then we have a new class of convex functions which is called as Godunova-Levin-Dragomir type of $\left(s_{1}, s_{2}\right)$ convex functions.

Definition 6. Let $s_{1}, s_{2} \in(0,1]$ be two real numbers. We say that $f: \mathcal{\ell} \rightarrow \mathbb{R}$ is an $\left(s_{1}, s_{2}\right)$-convex function, if

$$
f(t x+(1-t) y) \leq \frac{1}{t^{s_{1}}(1-t)^{s_{2}}} f(x)+\frac{1}{(1-t)^{s_{1}} t^{s_{2}}} f(y), \quad \forall x, y \in \mathcal{C}, t \in[0,1] .
$$

It is clear that these new classes of convex functions are quite general and include several new and previously known classes of convex functions as special cases.

The following results will be helpful in deriving our main results in this paper.

Lemma 1 ([13]). Let $f: \mathcal{l}=[a, b] \subset \mathbb{R} \rightarrow \mathbb{R}$ be a continuous function such that $f \in \mathscr{L}[a, b]$, then

$$
\int_{a}^{b}(u-a)^{\alpha}(b-u)^{\beta} f(u) \mathrm{d} u=(b-a)^{\alpha+\beta+1} \int_{0}^{1} t^{\alpha}(1-t)^{\beta} f((1-t) a+t b) \mathrm{d} t .
$$

Lemma 2 ([9]). Let $d^{\circ} \subset \mathbb{R} \rightarrow \mathbb{R}, a, b \in d^{\circ}$ with $a<b$ where $d^{\circ}$ is the interior of $d^{\circ}$. If $f^{(n)}$ exists on $\mathcal{d}^{\circ}$ and $f^{(n)} \in \mathscr{L}[a, b]$, then for $n \geq 1$, we have

$$
\frac{f(a)+f(b)}{2}-\frac{1}{b-a} \int_{a}^{b} f(x) \mathrm{d} x-\sum_{k=2}^{n-1} \frac{(k-1)(b-a)^{k}}{2(k+1) !} f^{(k)}(a)
$$




$$
=\frac{(b-a)^{n}}{2 n !} \int_{0}^{1} t^{n-1}(n-2 t) f^{(n)}(t a+(1-t) b) \mathrm{d} t .
$$

\section{MAIN RESUltS}

In this section we derive our main results.

Theorem 1. Let $f: d=[a, b] \subset \mathbb{R} \rightarrow \mathbb{R}$ be $a\left(h_{1}, h_{2}\right)$-convex function. If $f \in$ $\mathscr{L}[a, b]$, then for $h_{1}\left(\frac{1}{2}\right) h_{2}\left(\frac{1}{2}\right) \neq 0$, we have

$$
\frac{1}{2 h_{1}\left(\frac{1}{2}\right) h_{2}\left(\frac{1}{2}\right)} f\left(\frac{a+b}{2}\right) \leq \frac{1}{b-a} \int_{a}^{b} f(x) \mathrm{d} x \leq[f(a)+f(b)] \int_{0}^{1} h_{1}(t) h_{2}(1-t) \mathrm{d} t .
$$

Proof. Since $f$ is an $\left(h_{1}, h_{2}\right)$-convex function, for $x=t a+(1-t) b, y=$ $(1-t) a+t b$ and $t=\frac{1}{2}$, we have

$$
f\left(\frac{a+b}{2}\right) \leq h_{1}\left(\frac{1}{2}\right) h_{2}\left(\frac{1}{2}\right)[f(t a+(1-t) b)+f((1-t) a+t b)] .
$$

Integrating both sides of the above inequality with respect to $t$ on $[0,1]$, we have

$$
\frac{1}{2 h_{1}\left(\frac{1}{2}\right) h_{2}\left(\frac{1}{2}\right)} f\left(\frac{a+b}{2}\right) \leq \frac{1}{b-a} \int_{a}^{b} f(x) \mathrm{d} x .
$$

Also

$$
f(t a+(1-t) b) \leq h_{1}(t) h_{2}(1-t) f(x)+h_{1}(1-t) h_{2}(t) f(y) .
$$

Integrating both sides of the above inequality with respect to $t$ on $[0,1]$, we have

$$
\frac{1}{b-a} \int_{a}^{b} f(x) \mathrm{d} x \leq[f(a)+f(b)] \int_{0}^{1} h_{1}(t) h_{2}(1-t) \mathrm{d} t .
$$

On summation of inequalities (3.1) and (3.2) the proof is complete.

We now discuss a new special case of Theorem 1.

If $h_{1}(t)=t^{s_{1}}$ and $h_{2}=t^{s_{2}}$ in Theorem 1, then we have a result for Brecker type of $\left(s_{1}, s_{2}\right)$-convex functions.

Corollary 1. Let $f: \mathbb{d}=[a, b] \subset \mathbb{R} \rightarrow \mathbb{R}$ be a Brecker type of $\left(s_{1}, s_{2}\right)$-convex function. If $f \in \mathscr{L}[a, b]$, then for $s_{1}, s_{2} \in[0,1]$ we have

$$
\frac{1}{2^{1-s_{1}-s_{2}}} f\left(\frac{a+b}{2}\right) \leq \frac{1}{b-a} \int_{a}^{b} f(x) \mathrm{d} x \leq[f(a)+f(b)] \mathrm{B}\left(s_{1}+1, s_{2}+1\right) .
$$


If $h_{1}(t)=t^{-s_{1}}$ and $h_{2}=t^{-s_{2}}$ in Theorem 1 , then we have a result for GodunovaLevin-Dragomir type of $\left(s_{1}, s_{2}\right)$-convex functions.

Corollary 2. Let $f: d=[a, b] \subset \mathbb{R} \rightarrow \mathbb{R}$ be a Godunova-Levin-Dragomir type of $\left(s_{1}, s_{2}\right)$-convex function. If $f \in \mathscr{L}[a, b]$, then for $s_{1}, s_{2} \in[0,1]$, we have

$$
\frac{1}{2^{1+s_{1}+s_{2}}} f\left(\frac{a+b}{2}\right) \leq \frac{1}{b-a} \int_{a}^{b} f(x) \mathrm{d} x \leq[f(a)+f(b)] \mathbb{B}\left(1-s_{1}, 1-s_{2}\right) .
$$

Our next result is a lower bound for Hermite-Hadamard's inequality via product of two $\left(h_{1}, h_{2}\right)$-convex functions.

Theorem 2. Let $f, g: d=[a, b] \subset \mathbb{R} \rightarrow \mathbb{R}$ be two $\left(h_{1}, h_{2}\right)$-convex functions such that $h_{1}^{2}\left(\frac{1}{2}\right) h_{2}^{2}\left(\frac{1}{2}\right) \neq 0$. If $f g \in \mathscr{L}[a, b]$, then, we have

$$
\begin{aligned}
& \frac{1}{2 h_{1}^{2}\left(\frac{1}{2}\right) h_{2}^{2}\left(\frac{1}{2}\right)} f\left(\frac{a+b}{2}\right) g\left(\frac{a+b}{2}\right) \\
& -\left[M(a, b) \int_{0}^{1}\left[h_{1}(t) h_{2}(t) h_{1}(1-t) h_{2}(1-t)\right] \mathrm{d} t+N(a, b) \int_{0}^{1} h_{1}^{2}(t) h_{2}^{2}(1-t) \mathrm{d} t\right] \\
& \leq \frac{1}{b-a} \int_{0}^{1} f(x) g(x) \mathrm{d} x .
\end{aligned}
$$

where

$$
M(a, b)=f(a) g(a)+f(b) g(b),
$$

and

respectively.

$$
N(a, b)=f(a) g(b)+f(b) g(a),
$$

Proof. Since $f$ and $g$ are $\left(h_{1}, h_{2}\right)$-convex functions, so

$$
\begin{aligned}
& f\left(\frac{a+b}{2}\right) g\left(\frac{a+b}{2}\right) \\
& \leq h_{1}\left(\frac{1}{2}\right) h_{2}\left(\frac{1}{2}\right)[f(t a+(1-t) b)+f((1-t) a+t b)] \\
& \times h_{1}\left(\frac{1}{2}\right) h_{2}\left(\frac{1}{2}\right)[g(t a+(1-t) b)+g((1-t) a+t b)] \\
&= h_{1}^{2}\left(\frac{1}{2}\right) h_{2}^{2}\left(\frac{1}{2}\right)[f(t a+(1-t) b) g(t a+(1-t) b) \\
& f((1-t) a+t b) g((1-t) a+t b)+f(t a+(1-t) b) g((1-t) a+t b) \\
&+f((1-t) a+t b) g(t a+(1-t) b)]
\end{aligned}
$$




$$
\begin{aligned}
\leq & h_{1}^{2}\left(\frac{1}{2}\right) h_{2}^{2}\left(\frac{1}{2}\right)[f(t a+(1-t) b) g(t a+(1-t) b) \\
& +f((1-t) a+t b) g((1-t) a+t b) \\
& +\left[2 h_{1}(t) h_{2}(t) h_{1}(1-t) h_{2}(1-t)\right][f(a) g(a)+f(b) g(b)] \\
& \left.+\left[h_{1}^{2}(1-t) h_{2}^{2}(t)+h_{1}^{2}(t) h_{2}^{2}(1-t)\right][f(a) g(b)+f(b) g(a)]\right] .
\end{aligned}
$$

Integrating the above inequality with respect to $t$ on $[0,1]$, we have

$$
\begin{aligned}
& f\left(\frac{a+b}{2}\right) g\left(\frac{a+b}{2}\right) \\
& \leq 2 h_{1}^{2}\left(\frac{1}{2}\right) h_{2}^{2}\left(\frac{1}{2}\right)\left[\frac{1}{b-a} \int_{0}^{1} f(x) g(x) \mathrm{d} x\right. \\
& \left.\quad+M(a, b) \int_{0}^{1}\left[h_{1}(t) h_{2}(t) h_{1}(1-t) h_{2}(1-t)\right] \mathrm{d} t+N(a, b) \int_{0}^{1} h_{1}^{2}(t) h_{2}^{2}(1-t) \mathrm{d} t\right] .
\end{aligned}
$$

This implies

$$
\begin{aligned}
& \frac{1}{2 h_{1}^{2}\left(\frac{1}{2}\right) h_{2}^{2}\left(\frac{1}{2}\right)} f\left(\frac{a+b}{2}\right) g\left(\frac{a+b}{2}\right) \\
& \leq \frac{1}{b-a} \int_{0}^{1} f(x) g(x) \mathrm{d} x \\
& \quad+M(a, b) \int_{0}^{1}\left[h_{1}(t) h_{2}(t) h_{1}(1-t) h_{2}(1-t)\right] \mathrm{d} t+N(a, b) \int_{0}^{1} h_{1}^{2}(t) h_{2}^{2}(1-t) \mathrm{d} t .
\end{aligned}
$$

This completes the proof.

Next we discuss a new special case of Theorem 2.

If $h_{1}(t)=t^{s_{1}}$ and $h_{2}=t^{s_{2}}$ in Theorem 2, then we have a result for Breckner type of $\left(s_{1}, s_{2}\right)$-convex functions.

Corollary 3. Let $f, g: d=[a, b] \subset \mathbb{R} \rightarrow \mathbb{R}$ be two ( $\left(s_{1}, s_{2}\right)$-convex functions such that $s_{1}, s_{2} \in[0,1]$. If $f g \in \mathscr{L}[a, b]$, then we have

$$
\begin{aligned}
& \frac{1}{2^{1-2 s_{1}-2 s_{2}}} f\left(\frac{a+b}{2}\right) g\left(\frac{a+b}{2}\right) \\
& -\left[M(a, b) \mathbb{B}\left(s_{1}+s_{2}+1, s_{1}+s_{2}+1\right)+N(a, b) \mathbb{B}\left(2 s_{1}+1,2 s_{2}+1\right)\right]
\end{aligned}
$$




$$
\leq \frac{1}{b-a} \int_{0}^{1} f(x) g(x) \mathrm{d} x
$$

where $M(a, b)$ and $N(a, b)$ are given in (3.3) and (3.4) respectively.

If $h_{1}(t)=t^{-s_{1}}$ and $h_{2}=t^{-s_{2}}$ in Theorem 2, then we have a result for GodunovaLevin-Dragomir type of $\left(s_{1}, s_{2}\right)$-convex functions.

Corollary 4. Let $f, g: d=[a, b] \subset \mathbb{R} \rightarrow \mathbb{R}$ be two Godunova-Levin-Dragomir type of $\left(s_{1}, s_{2}\right)$-convex functions such that $s_{1}, s_{2} \in[0,1]$. If $f g \in \mathscr{L}[a, b]$, then we have

$$
\begin{aligned}
& \frac{1}{2^{1+2 s_{1}+2 s_{2}} f\left(\frac{a+b}{2}\right) g\left(\frac{a+b}{2}\right)} \\
& -\left[M(a, b) \mathbb{B}\left(1-s_{1}-s_{2}, 1-s_{1}-s_{2}\right)+N(a, b) \mathbb{B}\left(1-2 s_{1}, 1-2 s_{2}\right)\right] \\
& \leq \frac{1}{b-a} \int_{0}^{1} f(x) g(x) \mathrm{d} x
\end{aligned}
$$

where $M(a, b)$ and $N(a, b)$ are given in (3.3) and (3.4) respectively.

Our next result is the extension of the upper bound of Hermite-Hadamard type inequality via product of two $\left(h_{1}, h_{2}\right)$-convex functions.

Theorem 3. Left $f, g: d=[a, b] \rightarrow \mathbb{R}$ be two $\left(h_{1}, h_{2}\right)$-convex functions. If $f g \in$ $\mathscr{L}[a, b]$, then, we have

$$
\begin{aligned}
& \frac{1}{b-a} \int_{a}^{b} f(x) g(x) \mathrm{d} x \\
& \leq M(a, b) \int_{0}^{1} h_{1}^{2}(t) h_{2}^{2}(1-t) \mathrm{d} t+N(a, b) \int_{0}^{1} h_{1}(t) h_{2}(1-t) h_{1}(1-t) h_{2}(t) \mathrm{d} t,
\end{aligned}
$$

where $M(a, b)$ and $N(a, b)$ are given by (3.3) and (3.4) respectively.

Proof. Since $f$ and $g$ are $\left(h_{1}, h_{2}\right)$-convex functions, then

$$
f(t a+(1-t) b) \leq h_{1}(t) h_{2}(1-t) f(a)+h_{1}(1-t) h_{2}(t) f(b),
$$

and

$$
g(t a+(1-t) b) \leq h_{1}(t) h_{2}(1-t) g(a)+h_{1}(1-t) h_{2}(t) g(b) .
$$


Multiplying both sides of the above inequality and then integrating it with respect to $t$ on $[0,1]$, we have

$$
\begin{aligned}
& \int_{0}^{1} f(t a+(1-t) b) g(t a+(1-t) b) \mathrm{d} t \\
& \leq[f(a) g(a)+f(b) g(b)] \int_{0}^{1} h_{1}^{2}(t) h_{2}^{2}(1-t) \mathrm{d} t \\
& \quad+[f(a) g(b)+f(b) g(a)] \int_{0}^{1} h_{1}(t) h_{2}(1-t) h_{1}(1-t) h_{2}(t) \mathrm{d} t .
\end{aligned}
$$

This implies

$$
\begin{aligned}
& \frac{1}{b-a} \int_{a}^{b} f(x) g(x) \mathrm{d} x \\
& \leq M(a, b) \int_{0}^{1} h_{1}^{2}(t) h_{2}^{2}(1-t) \mathrm{d} t+N(a, b) \int_{0}^{1} h_{1}(t) h_{2}(1-t) h_{1}(1-t) h_{2}(t) \mathrm{d} t .
\end{aligned}
$$

This completes the proof.

The next result is a special case of Theorem 3 .

If $h_{1}(t)=t^{s_{1}}$ and $h_{2}=t^{s_{2}}$ in Theorem 3, then we have a result for Breckner type of $\left(s_{1}, s_{2}\right)$-convex functions.

Corollary 5. Let $f, g: d=[a, b] \rightarrow \mathbb{R}$ be two Breckner type of $\left(s_{1}, s_{2}\right)$-convex functions where $s_{1}, s_{2} \in[0,1]$. If $f g \in \mathscr{L}[a, b]$, then we have

$$
\begin{aligned}
& \frac{1}{b-a} \int_{a}^{b} f(x) g(x) \mathrm{d} x \\
& \leq M(a, b) \mathbb{B}\left(2 s_{1}+1,2 s_{2}+1\right)+N(a, b) \mathbb{B}\left(s_{1}+s_{2}+1, s_{1}+s_{2}+1\right),
\end{aligned}
$$

where $M(a, b)$ and $N(a, b)$ are given by (3.3) and (3.4) respectively.

If $h_{1}(t)=t^{-s_{1}}$ and $h_{2}=t^{-s_{2}}$ in Theorem 3 , then we have a result for GodunovaLevin-Dragomir type of $\left(s_{1}, s_{2}\right)$-convex functions. 
Corollary 6. Let $f, g: d=[a, b] \rightarrow \mathbb{R}$ be two Godunova-Levin-Dragomir type of $\left(s_{1}, s_{2}\right)$-convex functions where $s_{1}, s_{2} \in[0,1]$. If $f g \in \mathscr{L}[a, b]$, then we have

$$
\begin{aligned}
& \frac{1}{b-a} \int_{a}^{b} f(x) g(x) \mathrm{d} x \\
& \leq M(a, b) \mathbb{B}\left(1-2 s_{1}, 1-2 s_{2}\right)+N(a, b) \mathbb{B}\left(1-s_{1}-s_{2}, 1-s_{1}-s_{2}\right),
\end{aligned}
$$

where $M(a, b)$ and $N(a, b)$ are given by (3.3) and (3.4) respectively.

Theorem 4. Let $f: d=[a, b] \subset \mathbb{R} \rightarrow \mathbb{R}$ be a continuous function such that $f \in$ $\mathscr{L}[a, b]$. If $f$ is an $\left(h_{1}, h_{2}\right)$-convex function, then we have

$$
\int_{a}^{b}(u-a)^{\alpha}(b-u)^{\beta} f(u) \mathrm{d} u \leq(b-a)^{\alpha+\beta+1}\left[\psi_{1}(t) f(a)+\psi_{2}(t) f(b)\right],
$$

where

$$
\psi_{1}(t):=\int_{0}^{1} t^{\alpha}(1-t)^{\beta} h_{1}(1-t) h_{2}(t) \mathrm{d} t
$$

and

$$
\psi_{2}(t):=\int_{0}^{1} t^{\alpha}(1-t)^{\beta} h_{1}(t) h_{2}(1-t) \mathrm{d} t
$$

respectively.

Proof. Using Lemma 1 and the fact that $f$ is an $\left(h_{1}, h_{2}\right)$-convex function, we have

$$
\begin{aligned}
& \int_{a}^{b}(u-a)^{\alpha}(b-u)^{\beta} f(u) \mathrm{d} u \\
& =(b-a)^{\alpha+\beta+1} \int_{0}^{1} t^{\alpha}(1-t)^{\beta} f((1-t) a+t b) \mathrm{d} t \\
& \leq(b-a)^{\alpha+\beta+1} \int_{0}^{1} t^{\alpha}(1-t)^{\beta}\left[h_{1}(1-t) h_{2}(t) f(a)+h_{1}(t) h_{2}(1-t) f(b)\right] \mathrm{d} t \\
& =(b-a)^{\alpha+\beta+1}\left[\psi_{1}(t) f(a)+\psi_{2}(t) f(b)\right] .
\end{aligned}
$$

This completes the proof.

If $h_{1}(t)=t^{s_{1}}$ and $h_{2}(t)=t^{s_{2}}$ in Theorem 4, then we have 
Corollary 7. Let $f: d=[a, b] \subset \mathbb{R} \rightarrow \mathbb{R}$ be a continuous function such that $f \in$ $\mathscr{L}[a, b]$. If $f$ is a Breckner type of $\left(s_{1}, s_{2}\right)$-convex function, where $s_{1}, s_{2} \in[0,1]$, then we have

$$
\int_{a}^{b}(u-a)^{\alpha}(b-u)^{\beta} f(u) \mathrm{d} u \leq(b-a)^{\alpha+\beta+1}\left[\psi_{1}^{\prime}(t) f(a)+\psi_{2}^{\prime}(t) f(b)\right],
$$

where

$$
\psi_{1}^{\prime}(t):=\mathbb{B}\left(\alpha+s_{2}+, \beta+s_{1}+1\right)
$$

and

$$
\psi_{2}^{\prime}(t):=\mathbb{B}\left(\alpha+s_{1}+1, \beta+s_{2}+1\right),
$$

respectively.

If $h_{1}(t)=t^{-s_{1}}$ and $h_{2}(t)=t^{-s_{2}}$ in Theorem 4, then we have

Corollary 8. Let $f: d=[a, b] \subset \mathbb{R} \rightarrow \mathbb{R}$ be a continuous function such that $f \in$ $\mathscr{L}[a, b]$. If $f$ is a Godunova-Levin-Dragomir type of $\left(s_{1}, s_{2}\right)$-convex function, where $s_{1}, s_{2} \in[0,1]$, then we have

$$
\int_{a}^{b}(u-a)^{\alpha}(b-u)^{\beta} f(u) \mathrm{d} u \leq(b-a)^{\alpha+\beta+1}\left[\psi_{1}^{\prime \prime}(t) f(a)+\psi_{2}^{\prime \prime}(t) f(b)\right],
$$

where

$$
\psi_{1}^{\prime \prime}(t):=\mathbb{B}\left(\alpha-s_{2}+1, \beta-s_{1}+1\right),
$$

and

$$
\psi_{2}^{\prime \prime}(t):=\mathbb{B}\left(\alpha-s_{1}+1, \beta-s_{2}+1\right),
$$

respectively.

Theorem 5. Let $f: d=[a, b] \subset \mathbb{R} \rightarrow \mathbb{R}$ be a continuous function such that $f \in$ $\mathscr{L}[a, b]$. If $\mid f^{\frac{r}{r-1}}$ is a $\left(h_{1}, h_{2}\right)$-convex function, then we have

$$
\begin{aligned}
& \int_{a}^{b}(u-a)^{\alpha}(b-u)^{\beta} f(u) \mathrm{d} u \\
& \leq(b-a)^{\alpha+\beta+1} \mathbb{B}(r \alpha+1, r \beta+1)\left[\left\{|f(a)|^{\frac{r}{r-1}}+|f(b)|^{\frac{r}{r-1}}\right\} \int_{0}^{1} h_{1}(t) h_{2}(1-t) \mathrm{d} t\right]^{\frac{r-1}{r}} .
\end{aligned}
$$


Proof. Using Lemma 1, Holder's inequality and the fact that $|f|^{\frac{r}{r-1}}$ is an $\left(h_{1}, h_{2}\right)$ convex function, then

$$
\begin{aligned}
& \int_{a}^{b}(u-a)^{\alpha}(b-u)^{\beta} f(u) \mathrm{d} u \\
& \leq(b-a)^{\alpha+\beta+1}\left[\int_{0}^{1} t^{r \alpha}(1-t)^{r \beta} \mathrm{d} t\right]^{\frac{1}{r}}\left[\int_{0}^{1}|f((1-t) a+t b)|^{\frac{r}{r-1}} \mathrm{~d} t\right]^{\frac{r-1}{r}} \\
& \leq(b-a)^{\alpha+\beta+1} \mathbb{B}(r \alpha+1, r \beta+1) \\
& {\left[\int_{0}^{1}\left\{h_{1}(1-t) h_{2}(t)|f(a)|^{\frac{r}{r-1}}+h_{1}(t) h_{2}(1-t)|f(b)|^{\frac{r}{r-1}}\right\} \mathrm{d} t\right]^{\frac{r-1}{r}} } \\
& \leq(b-a)^{\alpha+\beta+1} \mathbb{B}(r \alpha+1, r \beta+1) \\
& {\left[\left\{|f(a)|^{\frac{r}{r-1}}+|f(b)|^{\frac{r}{r-1}}\right\} \int_{0}^{1} h_{1}(t) h_{2}(1-t) \mathrm{d} t\right]^{\frac{r-1}{r}} . }
\end{aligned}
$$

This completes the proof.

If $h_{1}(t)=t^{s_{1}}$ and $h_{2}(t)=t^{s_{2}}$ in Theorem 5, then we have

Corollary 9. Let $f: d=[a, b] \subset \mathbb{R} \rightarrow \mathbb{R}$ be a continuous function such that $f \in$ $\mathscr{L}[a, b]$. If $|f|^{\frac{r}{r-1}}$ is a Breckner type of $\left(s_{1}, s_{2}\right)$-convex function, where $s_{1}, s_{2} \in[0,1]$, then we have

$$
\begin{aligned}
& \int_{a}^{b}(u-a)^{\alpha}(b-u)^{\beta} f(u) \mathrm{d} u \\
& \leq(b-a)^{\alpha+\beta+1} \mathbb{B}(r \alpha+1, r \beta+1)\left[\left\{|f(a)|^{\frac{r}{r-1}}+|f(b)|^{\frac{r}{r-1}}\right\} \mathbb{B}\left(\alpha+s_{1}+1, \beta+s_{2}+1\right)\right]^{\frac{r-1}{r}} .
\end{aligned}
$$

If $h_{1}(t)=t^{-s_{1}}$ and $h_{2}(t)=t^{-s_{2}}$ in Theorem 5, then we have

Corollary 10. Let $f: d=[a, b] \subset \mathbb{R} \rightarrow \mathbb{R}$ be a continuous function such that $f \in$ $\mathscr{L}[a, b]$. If $|f|^{\frac{r}{r-1}}$ is a Godunova-Levin-Dragomir type of $\left(s_{1}, s_{2}\right)$-convex function, where $s_{1}, s_{2} \in[0,1]$, then we have

$$
\begin{aligned}
& \int_{a}^{b}(u-a)^{\alpha}(b-u)^{\beta} f(u) \mathrm{d} u \\
& \leq(b-a)^{\alpha+\beta+1} \mathbb{B}(1-r \alpha, 1-r \beta)\left[\left\{|f(a)|^{\frac{r}{r-1}}+|f(b)|^{\frac{r}{r-1}}\right\} \mathbb{B}\left(1-\alpha-s_{1}, 1-\beta-s_{2}\right)\right]^{\frac{r-1}{r}} .
\end{aligned}
$$


Theorem 6. Let $f: d=[a, b] \subset \mathbb{R} \rightarrow \mathbb{R}$ be a continuous function such that $f \in$ $\mathscr{L}[a, b]$. If $|f|^{r}$ is an $\left(h_{1}, h_{2}\right)$-convex function, then we have

$$
\begin{aligned}
& \int_{a}^{b}(u-a)^{\alpha}(b-u)^{\beta} f(u) \mathrm{d} u \\
& \leq(b-a)^{\alpha+\beta+1}[\mathbb{B}(\alpha+1, \beta+1)]^{\frac{r-1}{r}}\left[\psi_{1}(t)|f(a)|^{r}+\psi_{2}(t)|f(b)|^{r}\right]^{\frac{1}{r}},
\end{aligned}
$$

where $\psi_{1}(t)$ and $\psi_{2}(t)$ are given by (3.5) and (3.6) respectively.

Proof. Using Lemma 1, Holder's inequality and the fact that $|f|^{r}$ is an $\left(h_{1}, h_{2}\right)$ convex function, then

$$
\begin{aligned}
& \int_{a}^{b}(u-a)^{\alpha}(b-u)^{\beta} f(u) \mathrm{d} u \\
& \leq(b-a)^{\alpha+\beta+1}\left[\int_{0}^{1}(1-t)^{\alpha} t^{\beta} \mathrm{d} t\right]^{\frac{r-1}{r}}\left[\int_{0}^{1} t^{\alpha}(1-t)^{\beta}|f((1-t) a+t b)|^{r} \mathrm{~d} t\right]^{\frac{1}{r}} \\
& \leq(b-a)^{\alpha+\beta+1}[\mathrm{~B}(\alpha+1, \beta+1)]^{\frac{r-1}{r}} \\
& \quad \times\left[\int_{0}^{1} t^{\alpha}(1-t)^{\beta}\left[h_{1}(1-t) h_{2}(t)|f(a)|^{r}+h_{1}(t) h_{2}(1-t)|f(b)|^{r}\right] \mathrm{d} t\right]^{\frac{1}{r}} \\
& =(b-a)^{\alpha+\beta+1}[\mathbb{B}(\alpha+1, \beta+1)]^{\frac{r-1}{r}}\left[\psi_{1}(t)|f(a)|^{r}+\psi_{2}(t)|f(b)|^{r}\right]^{\frac{1}{r}} .
\end{aligned}
$$

This completes the proof.

If $h_{1}(t)=t^{s_{1}}$ and $h_{2}(t)=t^{s_{2}}$ in Theorem 6, then we have

Corollary 11. Let $f: d=[a, b] \subset \mathbb{R} \rightarrow \mathbb{R}$ be a continuous function such that $f \in$ $\mathscr{L}[a, b]$. If $|f|^{r}$ is a Breckner type of $\left(s_{1}, s_{2}\right)$-convex function, where $s_{1}, s_{2} \in[0,1]$, then we have

$$
\begin{aligned}
& \int_{a}^{b}(u-a)^{\alpha}(b-u)^{\beta} f(u) \mathrm{d} u \\
& \leq(b-a)^{\alpha+\beta+1}[\mathbb{B}(\alpha+1, \beta+1)]^{\frac{r-1}{r}}\left[\psi_{1}^{\prime}(t)|f(a)|^{r}+\psi_{2}^{\prime}(t)|f(b)|^{r}\right]^{\frac{1}{r}},
\end{aligned}
$$

where $\psi_{1}^{\prime}(t)$ and $\psi_{2}^{\prime}(t)$ are given by (3.7) and (3.8) respectively.

If $h_{1}(t)=t^{-s_{1}}$ and $h_{2}(t)=t^{-s_{2}}$ in Theorem 6 , then we have 
Corollary 12. Let $f: d=[a, b] \subset \mathbb{R} \rightarrow \mathbb{R}$ be a continuous function such that $f \in \mathscr{L}[a, b] . I f|f|^{r}$ is a Godunova-Levin-Dragomir type of $\left(s_{1}, s_{2}\right)$-convex function, where $s_{1}, s_{2} \in[0,1]$, then we have

$$
\begin{aligned}
& \int_{a}^{b}(u-a)^{\alpha}(b-u)^{\beta} f(u) \mathrm{d} u \\
& \leq(b-a)^{\alpha+\beta+1}[\mathrm{~B}(1-\alpha, 1-\beta)]^{\frac{r-1}{r}}\left[\psi_{1}^{\prime \prime}(t)|f(a)|^{r}+\psi_{2}^{\prime \prime}(t)|f(b)|^{r}\right]^{\frac{1}{r}},
\end{aligned}
$$

where $\psi_{1}^{\prime \prime}(t)$ and $\psi_{2}^{\prime \prime}(t)$ are given by (3.9) and (3.10) respectively.

Now using Lemma 2, we derive some new inequalities for $n$-times differentiable $\left(h_{1}, h_{2}\right)$-convex functions.

Theorem 7. Let $f: d^{\circ} \subset \mathbb{R} \rightarrow \mathbb{R}, a, b \in d^{\circ}$ with $a<b$ where $d^{\circ}$ is the interior of $d^{\circ}$. Also suppose $f^{(n)}$ exists on $\mathcal{d}^{\circ}$ and $f^{(n)} \in \mathscr{L}[a, b]$. If $\left|f^{(n)}\right|^{q}$ is an $\left(h_{1}, h_{2}\right)$ convex function, then for $n, q \geq 1$, we have

$$
\begin{aligned}
& \left|\frac{f(a)+f(b)}{2}-\frac{1}{b-a} \int_{a}^{b} f(x) \mathrm{d} x-\sum_{k=2}^{n-1} \frac{(k-1)(b-a)^{k}}{2(k+1) !} f^{(k)}(a)\right| \\
& \leq \frac{(b-a)^{n}}{2 n !}\left(\frac{n-1}{n+1}\right)^{1-\frac{1}{q}}\left(\theta_{1}(t)\left|f^{n}(a)\right|^{q}+\theta_{2}(t)\left|f^{n}(b)\right|^{q}\right)^{\frac{1}{q}},
\end{aligned}
$$

where

$$
\theta_{1}(t):=\int_{0}^{1} t^{n-1}(n-2 t) h_{1}(t) h_{2}(1-t) \mathrm{d} t,
$$

and

$$
\theta_{2}(t):=\int_{0}^{1} t^{n-1}(n-2 t) h_{1}(1-t) h_{2}(t) \mathrm{d} t
$$

respectively.

Proof. Using Lemma 2, property of modulus, power means inequality and the fact that $\left|f^{(n)}\right|^{q}$ is an $\left(h_{1}, h_{2}\right)$-convex function, we have

$$
\begin{aligned}
& \left|\frac{f(a)+f(b)}{2}-\frac{1}{b-a} \int_{a}^{b} f(x) \mathrm{d} x-\sum_{k=2}^{n-1} \frac{(k-1)(b-a)^{k}}{2(k+1) !} f^{(k)}(a)\right| \\
& =\left|\frac{(b-a)^{n}}{2 n !} \int_{0}^{1} t^{n-1}(n-2 t) f^{(n)}(t a+(1-t) b) \mathrm{d} t\right|
\end{aligned}
$$




$$
\begin{aligned}
\leq & \frac{(b-a)^{n}}{2 n !} \int_{0}^{1} t^{n-1}(n-2 t)\left|f^{(n)}(t a+(1-t) b)\right| \mathrm{d} t \\
\leq & \frac{(b-a)^{n}}{2 n !}\left(\int_{0}^{1} t^{n-1}(n-2 t) \mathrm{d} t\right){ }^{1-\frac{1}{q}}\left(\int_{0}^{1} t^{n-1}(n-2 t)\left|f^{(n)}(t a+(1-t) b)\right|^{q} \mathrm{~d} t\right)^{\frac{1}{q}} \\
\leq & \frac{(b-a)^{n}}{2 n !}\left(\frac{n-1}{n+1}\right)^{1-\frac{1}{q}} \\
& \times\left(\int_{0}^{1} t^{n-1}(n-2 t)\left[h_{1}(t) h_{2}(1-t)\left|f^{n}(a)\right|^{q}+h_{1}(1-t) h_{2}(t)\left|f^{n}(b)\right|^{q}\right] \mathrm{d} t\right)^{\frac{1}{q}} \\
= & \frac{(b-a)^{n}}{2 n !}\left(\frac{n-1}{n+1}\right)^{1-\frac{1}{q}}\left(\theta_{1}(t)\left|f^{n}(a)\right|^{q}+\theta_{2}(t)\left|f^{n}(b)\right|^{q}\right)^{\frac{1}{q}} .
\end{aligned}
$$

This completes the proof.

If $h_{1}(t)=t^{s_{1}}$ and $h_{2}(t)=t^{s_{2}}$ in Theorem 7, then we have

Corollary 13. Let $f: d^{\circ} \subset \mathbb{R} \rightarrow \mathbb{R}, a, b \in d^{\circ}$ with $a<b$ where $d^{\circ}$ is the interior of $d^{\circ}$. Also suppose $f^{(n)}$ exists on $d^{\circ}$ and $f^{(n)} \in \mathscr{L}[a, b]$. If $\left|f^{(n)}\right|^{q}$ is a Breckner type of $\left(s_{1}, s_{2}\right)$-convex function, then for $s_{1}, s_{2} \in[0,1]$ and $n, q \geq 1$, we have

$$
\begin{aligned}
& \left|\frac{f(a)+f(b)}{2}-\frac{1}{b-a} \int_{a}^{b} f(x) \mathrm{d} x-\sum_{k=2}^{n-1} \frac{(k-1)(b-a)^{k}}{2(k+1) !} f^{(k)}(a)\right| \\
& \leq \frac{(b-a)^{n}}{2 n !}\left(\frac{n-1}{n+1}\right)^{1-\frac{1}{q}}\left(\theta_{1}^{\prime}(t)\left|f^{n}(a)\right|^{q}+\theta_{2}^{\prime}(t)\left|f^{n}(b)\right|^{q}\right)^{\frac{1}{q}},
\end{aligned}
$$

where

$$
\theta_{1}^{\prime}(t):=n \mathbb{B}\left(n+s_{1}, s_{2}+1\right)-2 \mathbb{B}\left(n+s_{1}+1, s_{2}+1\right),
$$

and

$$
\theta_{2}^{\prime}(t):=n \mathbb{B}\left(n+s_{2}, s_{1}+1\right)-2 \mathbb{B}\left(n+s_{2}+1, s_{2}+1\right),
$$

respectively.

If $h_{1}(t)=t^{-s_{1}}$ and $h_{2}(t)=t^{-s_{2}}$ in Theorem 7 , then we have

Corollary 14. Let $f: d^{\circ} \subset \mathbb{R} \rightarrow \mathbb{R}, a, b \in d^{\circ}$ with $a<b$ where $d^{\circ}$ is the interior of $d^{\circ}$. Also suppose $f^{(n)}$ exists on $d^{\circ}$ and $f^{(n)} \in \mathscr{L}[a, b]$. If $\left|f^{(n)}\right|^{q}$ is a GodunovaLevin-Dragomir type of $\left(s_{1}, s_{2}\right)$-convex function, then for $s_{1}, s_{2} \in[0,1]$ and $n, q \geq 1$, 
we have

$$
\begin{aligned}
& \left|\frac{f(a)+f(b)}{2}-\frac{1}{b-a} \int_{a}^{b} f(x) \mathrm{d} x-\sum_{k=2}^{n-1} \frac{(k-1)(b-a)^{k}}{2(k+1) !} f^{(k)}(a)\right| \\
& \leq \frac{(b-a)^{n}}{2 n !}\left(\frac{n-1}{n+1}\right)^{1-\frac{1}{q}}\left(\theta_{1}^{\prime \prime}(t)\left|f^{n}(a)\right|^{q}+\theta_{2}^{\prime \prime}(t)\left|f^{n}(b)\right|^{q}\right)^{\frac{1}{q}}
\end{aligned}
$$

where

$$
\theta_{1}^{\prime \prime}(t):=n \mathbb{B}\left(n-s_{1}, 1-s_{2}\right)-2 \mathbb{B}\left(n-s_{1}+1,1-s_{2}\right),
$$

and

$$
\theta_{2}^{\prime \prime}(t):=n \mathbb{B}\left(n-s_{2}, 1-s_{1}\right)-2 \mathbb{B}\left(n-s_{2}+1,1-s_{2}\right),
$$

respectively.

Theorem 8. Let $f: d^{\circ} \subset \mathbb{R} \rightarrow \mathbb{R}, a, b \in d^{\circ}$ with $a<b$ where $d^{\circ}$ is the interior of $d^{\circ}$. Also suppose $f^{(n)}$ exists on $\mathcal{d}^{\circ}$ and $f^{(n)} \in \mathscr{L}[a, b]$. If $\left|f^{(n)}\right|^{P}$ is a $\left(h_{1}, h_{2}\right)$ convex function, then for $n, q \geq 1$, we have

$$
\begin{aligned}
& \left|\frac{f(a)+f(b)}{2}-\frac{1}{b-a} \int_{a}^{b} f(x) \mathrm{d} x-\sum_{k=2}^{n-1} \frac{(k-1)(b-a)^{k}}{2(k+1) !} f^{(k)}(a)\right| \\
& \leq \frac{(b-a)^{n}}{2 n(n-1)^{\frac{1}{q}}}\left(\phi_{1}(t)\left|f^{n}(a)\right|^{q}+\phi_{2}(t)\left|f^{n}(b)\right|^{q}\right)^{\frac{1}{q}},
\end{aligned}
$$

where

$$
\phi_{1}(t):=\int_{0}^{1} t^{q(n-1)}(n-2 t) h_{1}(t) h_{2}(1-t) \mathrm{d} t,
$$

and

$$
\phi_{2}(t):=\int_{0}^{1} t^{q(n-1)}(n-2 t) h_{1}(1-t) h_{2}(t) \mathrm{d} t
$$

respectively.

Proof. Using Lemma 2, property of modulus, Holder's inequality and the fact that $\left|f^{(n)}\right|^{q}$ is an $\left(h_{1}, h_{2}\right)$-convex function, we have

$$
\left|\frac{f(a)+f(b)}{2}-\frac{1}{b-a} \int_{a}^{b} f(x) \mathrm{d} x-\sum_{k=2}^{n-1} \frac{(k-1)(b-a)^{k}}{2(k+1) !} f^{(k)}(a)\right|
$$




$$
\begin{aligned}
= & \left|\frac{(b-a)^{n}}{2 n !} \int_{0}^{1} t^{n-1}(n-2 t) f^{(n)}(t a+(1-t) b) \mathrm{d} t\right| \\
\leq & \frac{(b-a)^{n}}{2 n !} \int_{0}^{1} t^{n-1}(n-2 t)\left|f^{(n)}(t a+(1-t) b)\right| \mathrm{d} t \\
\leq & \frac{(b-a)^{n}}{2 n !}\left(\int_{0}^{1}(n-2 t) \mathrm{d} t\right)^{1-\frac{1}{q}}\left(\int_{0}^{1} t^{q(n-1)}(n-2 t)\left|f^{(n)}(t a+(1-t) b)\right|^{q} \mathrm{~d} t\right)^{\frac{1}{q}} \\
\leq & \frac{(b-a)^{n}}{2 n !}(n-1)^{1-\frac{1}{q}} \\
& \times\left(\int_{0}^{1} t^{q(n-1)}(n-2 t)\left[h_{1}(t) h_{2}(1-t)\left|f^{n}(a)\right|^{q}+h_{1}(1-t) h_{2}(t)\left|f^{n}(b)\right|^{q}\right] \mathrm{d} t\right)^{\frac{1}{q}} \\
= & \frac{(b-a)^{n}}{2 n(n-1)^{\frac{1}{q}}}\left(\phi_{1}(t)\left|f^{n}(a)\right|^{q}+\phi_{2}(t)\left|f^{n}(b)\right|^{q}\right)^{\frac{1}{q}} .
\end{aligned}
$$

This completes the proof.

If $h_{1}(t)=t^{s_{1}}$ and $h_{2}(t)=t^{s_{2}}$ in Theorem 8, then we have

Corollary 15. Let $f: d^{\circ} \subset \mathbb{R} \rightarrow \mathbb{R}, a, b \in d^{\circ}$ with $a<b$ where $d^{\circ}$ is the interior of $\mathcal{d}^{\circ}$. Also suppose $f^{(n)}$ exists on $\mathcal{L}^{\circ}$ and $f^{(n)} \in \mathscr{L}[a, b]$. If $\left|f^{(n)}\right|^{P}$ is a Breckner type of $\left(s_{1}, s_{2}\right)$-convex function, then for $s_{1}, s_{2} \in[0,1]$ and $n, q \geq 1$, we have

$$
\begin{aligned}
& \left|\frac{f(a)+f(b)}{2}-\frac{1}{b-a} \int_{a}^{b} f(x) \mathrm{d} x-\sum_{k=2}^{n-1} \frac{(k-1)(b-a)^{k}}{2(k+1) !} f^{(k)}(a)\right| \\
& \leq \frac{(b-a)^{n}}{2 n(n-1)^{\frac{1}{q}}}\left(\phi_{1}^{\prime}(t)\left|f^{n}(a)\right|^{q}+\phi_{2}^{\prime}(t)\left|f^{n}(b)\right|^{q}\right)^{\frac{1}{q}},
\end{aligned}
$$

where

$$
\phi_{1}^{\prime}(t):=n \mathbb{B}\left(n q+s_{1}-q+1, s_{2}+1\right)-2 \mathbb{B}\left(n q+s_{1}-q+2, s_{2}+1\right),
$$

and

$$
\phi_{2}^{\prime}(t):=n \mathbb{B}\left(n q+s_{2}-q+1, s_{1}+1\right)-2 \mathbb{B}\left(n q+s_{2}-q+2, s_{1}+1\right),
$$

respectively.

If $h_{1}(t)=t^{-s_{1}}$ and $h_{2}(t)=t^{-s_{2}}$ in Theorem 8 , then we have 
Corollary 16. Let $f: d^{\circ} \subset \mathbb{R} \rightarrow \mathbb{R}, a, b \in d^{\circ}$ with $a<b$ where $d^{\circ}$ is the interior of $d^{\circ}$. Also suppose $f^{(n)}$ exists on $d^{\circ}$ and $f^{(n)} \in \mathscr{L}[a, b]$. If $\left|f^{(n)}\right|^{P}$ is a GodunovaLevin-Dragomir type of $\left(s_{1}, s_{2}\right)$-convex function, then for $s_{1}, s_{2} \in[0,1]$ and $n, q \geq 1$, we have

$$
\begin{aligned}
& \left|\frac{f(a)+f(b)}{2}-\frac{1}{b-a} \int_{a}^{b} f(x) \mathrm{d} x-\sum_{k=2}^{n-1} \frac{(k-1)(b-a)^{k}}{2(k+1) !} f^{(k)}(a)\right| \\
& \leq \frac{(b-a)^{n}}{2 n(n-1)^{\frac{1}{q}}}\left(\phi_{1}^{\prime \prime}(t)\left|f^{n}(a)\right|^{q}+\phi_{2}^{\prime \prime}(t)\left|f^{n}(b)\right|^{q}\right)^{\frac{1}{q}},
\end{aligned}
$$

where

$$
\phi_{1}^{\prime \prime}(t):=n \mathbb{B}\left(n q-s_{1}-q+1,1-s_{2}\right)-2 \mathbb{B}\left(n q-s_{1}-q+2,1-s_{2}\right),
$$

and

$$
\phi_{2}^{\prime \prime}(t):=n \mathbb{B}\left(n q-s_{2}-q+1,1-s_{1}\right)-2 \mathbb{B}\left(n q-s_{2}-q+2,1-s_{1}\right),
$$

respectively.

\section{CONCLUSION}

In this paper, we have introduced a new extension of convex functions which is called as $\left(h_{1}, h_{2}\right)$-convex functions. We have noticed that it contains some new and known classes of convex functions among one of those is $h$-convex functions. We have derived several new generalizations of Hermite-Hadamard type inequalities via $\left(h_{1}, h_{2}\right)$-convex functions. We have also discussed some of new special cases which can be easily deduced from our main results. It is expected that the interested readers may further explore the property of $\left(h_{1}, h_{2}\right)$-convexity of functions.

\section{ACKNOWLEDGEMENTS}

This research is supported by HEC SRGP project No: 21-985/SRGP/R $\& \mathrm{D} / \mathrm{HEC} / 2016$.

\section{REFERENCES}

[1] W. W. Breckner, "Stetigkeitsaussagen fiir eine klasse verallgemeinerter convexer funktionen in topologischen linearen," Raumen. Pupl. Inst. Math., vol. 23, pp. 13-20, 1978.

[2] G. Cristescu and L. Lupsa, Non-connected Convexities and Applications. Kluwer Academic Publishers, Dordrecht, Holland, 2002.

[3] G. Cristescu, M. A. Noor, and M. U. Awan, "Bounds of the second degree cumulative frontier gaps of functions with generalized convexity," Carpathian J. Math., vol. 31, pp. 173-180, 2015.

[4] S. S. Dragomir, "Inequalities of jensen type for $\varphi$-convex functions," Fasciculi Mathematici, 2015.

[5] S. S. Dragomir and R. P. Agarwal, "Two inequalities for differentiable mappings and applications to special means of real numbers and to trapezoidal formula," Appl. Math. Lett., vol. 11, pp. 91-95, 1998. 
[6] S. S. Dragomir and C. E. M. Pearce, Selected topics on Hermite-Hadamard inequalities and applications. Victoria University, Australia, 2000.

[7] S. S. Dragomir, J. Pecaric, and L. E. Persson, "Some inequalities of hadamard type," Soochow J. Math., vol. 21, pp. 335-341, 1995.

[8] E. K. Godunova and V. I. Levin, "Neravenstva dlja funkcii sirokogo klassa, soderzascego vypuklye, monotonnye i nekotorye drugie vidy funkii," Vycislitel. Mat. i. Fiz. Mezvuzov. Sb. Nauc. Trudov, MGPI, Moskva, pp. 138-142, 1985.

[9] D.-Y. Hwang, "Some inequalities for $n$-time differentiable mappings and applications," Kyungpook Math. J., vol. 43, pp. 335-343, 2003.

[10] S. K. Khattri, "Three proofs of the inequality $e<\left(1+\frac{1}{n}\right)^{n+0.5}$," Amer. Math. Monthly, vol. 117, pp. 273-277, 2010.

[11] W. Liu, "New integral inequalities involving beta function via p-convexity," Miskolc Math. Notes, vol. 15 , pp. 585-591, 2014.

[12] M. A. Noor, M. U. Awan, and K. I. Noor, "Some new bounds of the quadrature formula of gaussjacobi type via ( $p, q)$-preinvex functions," Appl. Math. Inform. Sci. Lett., vol. 5, pp. 51-56, 2017.

[13] M. E. Ozdemir, E. Set, and M. Alomari, "Integral inequalities via several kinds of convexity," Creat. Math, Inform., vol. 20, pp. 62-73, 2011.

[14] C. E. M. Pearce and J. Pecaric, "Inequalities for differentiable mappings with application to special means and quadrature formula," Appl. Math. Lett., vol. 13, pp. 51-55, 2000.

[15] M. Z. Sarikaya, A. Saglam, and H. Yildrim, "On some hadamard-type inequalities for $h$-convex functons," J. Math. Inequal., vol. 2, pp. 335-341, 2008.

[16] E. Set, "New inequalities of ostrowski type for mappings whose derivatives are $s$-convex in the second sense via fractional integrals," Comput. \& Math. Appl., vol. 63, pp. 1147-1154, 2012.

[17] M. Tunc, E. Gov, and U. Sanal, "On tgs-convex function and their inequalities," Facta universitatis (NIS) Ser. Math. Inform., vol. 30, pp. 679-691, 2015.

[18] S. Varošanec, “On h-convexity,” J. Math. Anal. Appl., vol. 326, pp. 303-311, 2007.

Authors' addresses

Muhammad Uzair Awan

Department of Mathematics, GC University, Faisalabad, Pakistan

E-mail address: awan.uzair@gmail.com

Muhammad Aslam Noor

Department of Mathematics, COMSATS Institute of Information Technology, Islamabad, Pakistan

E-mail address: noormaslamegmail.com

Khalida Inayat Noor

Department of Mathematics, COMSATS Institute of Information Technology, Islamabad, Pakistan

E-mail address: khalidanoor@hotmail.com

Awais Gul Khan

Department of Mathematics, GC University, Faisalabad, Pakistan

E-mail address: awaisgulkhan@gmail.com 